\title{
FATHER TIME. I. DOES THE COSMIC MICROWAVE BACKGROUND RADIATION PROVIDE A UNIVERSAL ARROW OF TIME ?
}

\author{
T K Rai Dastidar* \\ Atomic 8 Molecular Physics Group, Department of Materials Science, \\ Indian Association for the Cultivation of Science, \\ Calcutta 700 032, India
}

\begin{abstract}
It was demonstrated in a recent paper (Mod. Phys. Lett. A13, 1265 (1998) ; hepth/9902020) that the existence of a non-thermodynamic arrow of time at the atomic level is a fundamental requirement for conservation of energy in matter-radiation interaction. In this paper we show that the Cosmic Microwave Background Radiation can provide this time's arrow to all particles in the universe that are acted on by the electromagnetic field.
\end{abstract}

In quantum field theory, the electromagnetic field is known to make the Lagrangian density of, e.g., a complex scalar field covariant under the U(1) local gauge transformation (GT) $\exp (-i e \Lambda(x))$, where $x \equiv(\mathbf{r}, t)$. (Note that a non-dynamical, pure gauge field can do this too [1]). We showed recently [2] that under a generalisation of U(1) to a non-local gauge transformation ${ }^{\mathrm{a}}$

$$
\exp (-i e \Lambda(x)) \rightarrow \exp \left(-i e \Lambda|x\rangle\left\langle x^{\prime}\right|\right)
$$

the covariance property of the Lagrangian is maintained, provided we replace the ordinary electromagnetic four-potential $A_{\mu}(x)$ by a non-local potential $\mathcal{A}_{\mu}|x\rangle\left\langle x^{\prime}\right|$, which transforms under the above non-local GT as

$$
\mathcal{A}_{\mu}|x\rangle\left\langle x^{\prime}\left|\rightarrow \mathcal{A}_{\mu}\right| x\right\rangle\left\langle x^{\prime}\left|+\partial_{\mu} \Lambda\right| x\right\rangle\left\langle x^{\prime}\left|+d_{\mu} \Lambda\right| x\right\rangle\left\langle x^{\prime}\right|
$$

where $\partial_{\mu} \equiv \partial / \partial x^{\mu}, d_{\mu} \equiv \partial / \partial x^{\mu}$. A phenomenological form of the interaction of matter with this non-local QED had been given quite a few years ago [3]. The non-local electromagnetic field tensor given by

$$
\mathcal{F}^{\mu \nu} \equiv \mathcal{F}^{\mu \nu}|x\rangle\left\langle x^{\prime}\left|=\left(\partial^{\mu}+d^{\mu}\right) \mathcal{A}^{\nu}\right| x\right\rangle\left\langle x^{\prime}\left|-\left(\partial^{\nu}+d^{\nu}\right) \mathcal{A}^{\mu}\right| x\right\rangle\left\langle x^{\prime}\right|
$$

* Electronic address : mstkrd@mahendra.iacs.res.in

a In order to remove possible misunderstandings regarding this notation in [2], we have given a detailed operational meaning of this non-local GT in Appendix A. 
remains invariant under the gauge transformation (2) if and only if

$$
\left[\partial^{\mu}, d^{\nu}\right]=0
$$

The relation (4) may therefore be considered as the necessary and sufficient condition for the non-local gauge transformation (1) to be a symmetry of the field. (Actually, if the relation (4) is satisfied, this new symmetry becomes theoretically self-consistent; we are going to address very shortly the question whether this symmetry is, in fact, obeyed by nature.) Before proceeding further, let us recall the three important results obtained in [2] :

i) As a consequence of matter-(non-local radiation) interaction, a correlated two-photon absorption linear in intensity occurs. (This had already been predicted in [3].) Direct experimental evidence for such two-photon absorption exists [4].

ii) The principle of causality emerges as a necessary condition for energy conservation in such correlated two-photon absorption processes ; out of the entire conceivable range of non-local temporal correlations (which spans both the past and the future), only the past ensures energy conservation in matter-radiation interaction events of the above kind, thus defining a non-thermodynamic arrow of time in the quantum (atomic) level.

iii) The self-consistency relation (4), which implies that the two space-time points $x, x^{\prime}$ are completely independent (i.e. need not lie within the light cone of one another), shows that the non-local correlation must be of the Einstein-Podolsky-Rosen (EPR)type. It was shown in [2] that this EPR-like character of the non-local correlation in the new QED is another necessary condition for energy conservation.

Note that the results (i) and (iii) address the question mentioned after equation (4). The result (iii) is very closely linked with the result (ii), and the present paper hinges on the arrow of time given in the latter ; for completeness we re-derive the results (ii) and (iii) in the appendix B.

The purpose of the present paper is to show that the cosmic microwave background radiation (CMBR) provides a via media for imparting each and every particle (which is acted on by an electromagnetic field) in the universe with this arrow of time. We proceed to demonstrate this by recalling from a recent work [5] that the interaction of matter with CMBR fits into the nonlocal picture as laid out in [2], and thus allows for correlated twophoton absorption by all particles, whereupon the time's arrow is established by energy conservation.

The substance of [2] is that if a radiation field (either as generated, or as detected by atomic electrons) enjoys a large second-order coherence — in other words, two photons far apart in space can be coherently absorbed by the atomic electrons within a phase difference $\Delta \phi=\omega \delta t \ll 1$ ( $\delta t$ being the time interval between the two photon-absorption events) - then the field is describable by the nonlocal QED. In practice, this translates to the condition that the photon density be significantly higher than the absorber (electron) density. (Of course, if the effect is to be measurable, the electron density must at the same time be high enough to make the observation of the two-photon absorption process experimentally viable.) Some typical matrix elements for such correlated two-photon absorption 
by atoms have been worked out in [2]. The question is : under what circumstances can this coherence condition be satisfied in practice ? Two suitable laboratory settings have already been described in [2], namely, (i) a laser field so intense that the average photon density is much larger than the electron density, and (ii) a squeezed light field with phase-correlated photon pairs, e.g. signal and idler photons. Very recently we have shown [5] that a third, cosmological setting is provided by this universe through the via media of the cosmic microwave background radiation (CMBR). Indeed, we can call the CMBR the "Lowest common denominator" from the viewpoint of this paper, in the sense that it influences each particle of the universe capable of interacting with an electromagnetic field. Note that, in the entire universe, the overall photon density is several orders of magnitude higher than the baryon or lepton density. As such, if we remember that the CMBR is incident from all directions, it is obvious that so long as the matter density is tenuous enough (so that the photon density remains higher than the matter density), two distinct photon absorption events can always take place at two arbitrary space points so that the above second-order coherence condition is satisfied. For example, it is easy to see that if two millimetre-wave photons are absorbed within a gap of $\sim 0.1-1 \mathrm{ps}$, then the phase gap between them is $\ll 1[6]$. The difference between the laboratory settings and the cosmological setting is that, whereas in the former the spatial distance between the two distinct (photon absorption) events is of the order of atomic/molecular dimensions, in the latter the said distance can be of macroscopic dimensions.

In [5] the consequences of such a correlated two-photon absorption loss upon the continuous spectrum of a blackbody radiation travelling through gaseous matter were worked out. It was shown there that the blackbody radiation energy density $E(\nu)$ at a frequency $\nu$ which, in the absence of any absorbing matter, is given by the Planck formula, would now be reduced to

$$
G(\nu)=E(\nu)-F(\nu), \quad F(\nu)=\alpha E(\nu / 2)
$$

where $\alpha$ is an effective correlated two-photon absorption factor for the intervening medium. For the present we need not go into the detailed dynamics needed for calculating $\alpha$; we only note that it will depend, among other things, on the density of the absorber. Detailed expressions for $E(\nu), F(\nu)$ and $G(\nu)$ have been given in [5]. It was shown that the theory predicts an apparent shift in the "blackbody temperature" of the radiation travelling through the gas, and an experimental test for checking this prediction was suggested.

Is there any observational evidence that such a two-photon absorption from the CMBR really does take place anywhere in the universe? There is, as has been demonstrated in [5]. To recapitulate briefly, such a two-photon absorption loss superimposed upon the spectrum of the CMBR travelling through interstellar and intergalactic matter manifests itself as an apparent deviation of the spectrum from the Planck formula - the magnitude of the deviation depends, of course, on the value of the absorption factor $\alpha$ - and hence this apparent deviation should be more "visible" when the CMBR is observed from within some dense gaseous atmosphere than from within more tenuous atmospheres. We now draw the reader's attention to the balloon-borne measurements of the cosmic microwave spectrum carried out by Woody and Richards [7] and the measurements carried out in the COBE 
(Cosmic Background Explorer) satellite [8], which fit into these two categories admirably. The spectrum observed by Woody and Richards [7] (see Fig. 2 therein) shows a distinct dip below the Planck curve over extended regions towards the right of the spectral peak, the maximum deviation occuring around twice the peak frequency - exactly as implied by the eqn. (5) - whereas in the $C O B E$ measurements the deviations from the Planck curve are much more miniscule.

To avoid misgivings that we are jumping to conclusions, we emphasize two points. First, whether such a deviation from the Planck distribution as predicted by our theory does occur at all is a question that can only be settled by experiment; one possible experimental test has been suggested in [5]. Secondly, the viability of such a correlated twophoton absorption process under conditions of Woody and Richards' balloon measurements has also been established in [5]. Therefore, subject to the condition that our prediction is experimentally verified, we can claim that the CMBR travelling through tenuous matter can satisfy the condition for second-order coherence and hence, as laid down by the nonlocal QED, is capable of giving rise to correlated two-photon absorption throughout the universe - thus offering a very simple picture of the arrow of time in the entire universe at the quantum (atomic) level as a necessary condition for energy conservation.

Our picture does not depend on any particular cosmological model. However, if one couples it to the Big Bang model, it would appear that the time's arrow (and hence causality) were set in motion as soon as the universe started off - the latter "creating", as it were, its own flow of time as well as its own physical laws as soon as it was born. Appealing as the picture is, it is still incomplete ; until the non-local gauge symmetry of the universe can be extended beyond eqn. (2) to cover the entire range of electroweak interactions, our theory remains confined only to those constituents of the universe that are acted on by the electromagnetic field and hence cannot embrace neutrinos, which are generally supposed to make up the cold dark matter in the universe. Work is in progress towards this goal.

The author is indebted to Dr Krishna Rai Dastidar, Department of Spectroscopy for useful discussions and to Dr J Chakraborti, Department of Theoretical Physics for his interest. A correspondence with Prof. Alex Dalgarno has helped to clarify certain ideas. 


\section{Appendix A}

In the earlier papers (MPLA 13, 1265 (1998) and 14, 1193 (1999)) we had introduced the non-local Gauge Transformation operator $T=\exp \left(-i e \Lambda|x\rangle\left\langle x^{\prime}\right|\right)$ simply as a shorthand notation for an integral operator. To avoid any possible misunderstandings arising out of our notation, we further clarify below the operational meaning of such non-local integral operators acting on an arbitrary field $\phi(x)$. First of all, $|x\rangle,\left\langle x^{\prime}\right|$ are not Dirac ket and bra vectors ; and in general, the operational meaning of any non-local (integral) operators $P|x\rangle\left\langle x^{\prime}|, Q| x\right\rangle\left\langle x^{\prime}\right|$ is given by the expressions

$$
P \phi \equiv \int P\left(x, x^{\prime}\right) \phi\left(x^{\prime}\right) d^{4} x^{\prime}, \quad P Q \phi \equiv \iint P\left(x, x^{\prime}\right) Q\left(x^{\prime}, x^{\prime \prime}\right) \phi\left(x^{\prime \prime}\right) d^{4} x^{\prime} d^{4} x^{\prime \prime}
$$

Thus the effect of the non-local gauge transformation $T$ on $\phi$ is given by

$$
T \phi \equiv \int e^{-i e \Lambda\left(x, x^{\prime}\right)} \phi\left(x^{\prime}\right) d^{4} x^{\prime}
$$

where $\Lambda\left(x, x^{\prime}\right)$ can be any arbitrary scalar function of $x$ and $x^{\prime}$. Thus, for infinitesimal $\Lambda$, we have $\delta \phi=T \phi-\phi=-i e \int \Lambda\left(x, x^{\prime}\right) \phi\left(x^{\prime}\right) d^{4} x^{\prime}$. One at once notes the parallelism with the Lippmann-Schwinger equation in the first order :

$$
\begin{aligned}
\Psi & =\left(1+G_{0} V\right) \psi \\
& =\psi(\vec{r})+\int G_{0}\left(\vec{r}, \vec{r}^{\prime}\right) V\left(\vec{r}^{\prime}\right) \psi\left(\vec{r}^{\prime}\right) d^{3} r^{\prime}
\end{aligned}
$$

where $\psi$ is an unperturbed state, $\Psi$ is a scattering state, $G_{0}\left(\vec{r}, \vec{r}^{\prime}\right)$ is the non-local Green's function $\left(E-H_{0}\right)^{-1}$ and $V$ is the scattering potential.

Similarly, the newly defined derivative

$$
\begin{aligned}
D_{\mu} \phi & =\partial_{\mu} \phi(x)+d_{\mu} \phi(x)+i e \mathcal{A}_{\mu}|x\rangle\left\langle x^{\prime}\right| \phi \\
& =\partial_{\mu} \phi(x)+d_{\mu} \phi(x)+i e \int \mathcal{A}_{\mu}\left(x, x^{\prime}\right) \phi\left(x^{\prime}\right) d^{4} x^{\prime}
\end{aligned}
$$

$\left(d_{\mu}=\partial / \partial x^{\prime \mu}\right.$, as defined in the Appendix A of the earlier paper) is covariant, provided $\mathcal{A}$ transforms under this gauge transformation as

$$
\mathcal{A}_{\mu} \phi \rightarrow \mathcal{A}_{\mu} \phi+\int\left[\partial_{\mu} \Lambda\left(x, x^{\prime}\right)\right] \phi\left(x^{\prime}\right) d^{4} x^{\prime}+\int\left[d_{\mu} \Lambda\left(x, x^{\prime}\right) \phi\left(x^{\prime}\right)\right] d^{4} x^{\prime}
$$

as may be easily checked using eqn. (A1). (Of course, for local $\phi$, the second term on the right side of $D_{\mu} \phi$ vanishes.) All the results derived in the earlier papers and the present paper are obtained without assuming any specific functional form of the quantities $\Lambda\left(x, x^{\prime}\right)$ and $\mathcal{A}\left(x, x^{\prime}\right)$. 


\section{Appendix B}

We briefly recapitulate here, following [2], how the time's arrow and the EPR-like character of the non-local correlation in the new QED appear in the theory as necessary conditions for conservation of energy in the atomic scale. A matrix element for matterradiation interaction with the electromagnetic field potential $\mathcal{A}\left(x, x^{\prime}\right)$ (which transforms as eqn. (2)) is typically of the form

$$
M_{f i}(t) \propto\left\langle\psi_{f}(x), n_{f}\left|\hat{p} \cdot \mathcal{A}\left(x, x^{\prime}\right)\right| \psi_{i}\left(x^{\prime}\right), n_{i}\right\rangle
$$

the integration running over $d^{3} r$ and $d^{4} x^{\prime}$. Special relativity requires that the time integral over $d t^{\prime}$ should run from $-\infty$ to $(t-\rho / c)$ [where $\left.\rho=\left|\mathbf{r}-\mathbf{r}^{\prime}\right|\right]$ for the retarded interaction, and from $(t+\rho / c)$ to $+\infty$ for the advanced interaction. After Fourier expanding $\mathcal{A}$ in two sets of photon modes (see, e.g. [9]), the time integration over $d t$ gives us the energyconserving delta-function $\delta\left(E_{f}, E_{i}+\hbar \omega+\hbar \omega^{\prime}\right)$ in the two-photon absorption process (see below). If we agree to define that the atom has been excited, i.e. the matrix element has come into existence, at the time $t=0$, then it is at once obvious that the the time integral over $d t$ can only run from 0 to $\infty$, giving finally a double integral to be chosen from

$$
\int_{0}^{\infty} f(t) d t\left(\int_{-\infty}^{t-\rho / c} g\left(t^{\prime}\right) d t^{\prime}, \quad \int_{t+\rho / c}^{\infty} g\left(t^{\prime}\right) d t^{\prime}\right)
$$

where $f(t)=\exp \left[\frac{i}{\hbar}\left(E_{f} t-\hbar \omega t\right)\right], g\left(t^{\prime}\right)=\exp \left[-\frac{i}{\hbar}\left(E_{i} t^{\prime}+\hbar \omega^{\prime} t^{\prime}\right)\right]$. It is obvious that the retarded interaction gives the required $\delta$-function for energy conservation, while the advanced interaction fails to do so. Thus we are constrained to limit the non-local temporal correlation to the past only; the fundamental principle of energy conservation has given us an arrow of time, i.e. the principle of causality, as a corollary.

However, one must not lose sight of a very important point : $x$ and $x^{\prime}$ need not be signal-linked. This is contrary to the spirit of special relativity, and requires an explanation. We note that if $x$ and $x^{\prime}$ are constrained to be so linked, then for $\rho>c t$ (remember that $\mathbf{r}$ and $\mathbf{r}^{\prime}$ extend over all space) the upper limit of the retarded $t^{\prime}$-integral becomes negative and the integral vanishes, leading to non-conservation of energy ! We are therefore obliged

to re-write the retarded integral as $\int_{-\infty}^{t} g\left(t^{\prime}\right) d t^{\prime}$. This is the physical reason why the nonlocal correlation must be of the "spooky" EPR-type ; the fact that this characteristic is required for theoretical self-consistency has already been mentioned in connection with eqn. (4). 


\section{References}

1. T K Rai Dastidar and Krishna Rai Dastidar, Mod. Phys. Lett. A10, 1843 (1995) ; Nuovo Cimento 109B, 1115 (1994)

2. T K Rai Dastidar and Krishna Rai Dastidar, Mod. Phys. Lett. A13, 1265 (1998) ; Errata A13, 2247 (1998) ; hep-th/9902020

3. (a) T K Rai Dastidar and Krishna Rai Dastidar, Abstracts of XI Int. Conf. At. Phys. (Paris, 1988), p. I-23 ; (b) Krishna Rai Dastidar in "Advances in Atomic and Molecular Physics", ed. M S Z Chaghtai (Today's and Tomorrow's Publishers, New Delhi, 1992) p. 49.

4. N P Georgiades, E S Polzik, K Edamatsu, H J Kimble and A S Parkins, Phys. Rev. Lett. 75, 3426 (1995)

5. T K Rai Dastidar, Mod. Phys. Lett. A 14, 1193 (1999) ; quant-ph/9903043

6. Remember that there need not be any causal link between the two events - indeed, in most cases there cannot be. Two events at two space-time points $x, x^{\prime}$ can be phasematched without any causal correlation, i.e. without the events' "knowing" about each other. As a matter of fact, this furnishes the clue to the EPR-like character of the nonlocal QED.

7. D P Woody and P L Richards, Phys. Rev. Lett. 42, 925 (1979)

8. J C Mather et al, Astrophys. J. 420, 439 (1994)

9. I M Gel'fand and G E Shilov, Generalised Functions (Acad. Press, 1964) Vol. 1, Chap. 2, eq. 1.3(1) 